PROCEEDINGS OF THE

AMERICAN MATHEMATICAL SOCIETY

Volume 135, Number 7, July 2007, Pages 2301-2307

S 0002-9939(07)08818-1

Article electronically published on March 2, 2007

\title{
THE GEOGRAPHY OF SYMPLECTIC 4-MANIFOLDS WITH AN ARBITRARY FUNDAMENTAL GROUP
}

\author{
JONGIL PARK
}

(Communicated by Daniel Ruberman)

\begin{abstract}
In this article, for each finitely presented group $G$, we construct a family of minimal symplectic 4-manifolds with $\pi_{1}=G$ which cover most lattice points $(x, \mathbf{c})$ with $x$ large in the region $0 \leq \mathbf{c}<9 x$. Furthermore, we show that all these 4 -manifolds admit infinitely many distinct smooth structures.
\end{abstract}

\section{INTRODUCTION}

Applications of gauge theory to symplectic 4-manifolds have led to many remarkable results. For example, C. Taubes proved that every minimal symplectic 4-manifold with $b_{2}^{+}>1$ satisfies $c_{1}^{2} \geq 0$ T]. Topologists were able to prove that most lattice points $(x, \mathbf{c})$ satisfying $0 \leq \mathbf{c}<9 x$ are realized as chern numbers $\left(\chi, c_{1}^{2}\right)$ of simply connected minimal symplectic 4-manifolds, and most known simply connected minimal symplectic 4-manifolds with $b_{2}^{+}$large enough admit infinitely many distinct symplectic structures ([FS], GS], P1]- P3] $)$. Note that $\chi=\frac{\sigma+e}{4}$ and $c_{1}^{2}=3 \sigma+2 e$, where $\sigma$ and $e$ denote the signature and the Euler characteristic of a given 4-manifold. But little is known in the non-simply connected case.

The aim of this paper is to extend the results above partially to the non-simply connected case. Explicitly we have

Theorem 1.1. For each finitely generated group $G$, there are constants $r_{G}$ and $t_{G}$ such that every lattice point $(x, \mathbf{c})$ satisfying $0 \leq \mathbf{c} \leq r_{G} x$ and $x \geq t_{G}$ is realized as $\left(\chi, c_{1}^{2}\right)$ of a minimal symplectic 4-manifold with $\pi_{1}=G$ which admits infinitely many distinct smooth structures. Furthermore, the constant $r_{G}$ can be chosen so that it is close to 9 .

Remarks 1. R. Gompf first constructed a new family of symplectic 4-manifolds with any prescribed fundamental group $\mathrm{G}$, and recently S. Baldridge and P. Kirk constructed a family of symplectic 4-manifolds with an arbitrary fundamental group near the Bogomolov-Miyaoka-Yau line BK2. Theorem 1.1 above addresses the uniqueness of smooth structures on such 4-manifolds as well as the existence of such 4-manifolds.

Received by the editors March 23, 2006.

2000 Mathematics Subject Classification. Primary 57R17, 57R57; Secondary 57N13.

Key words and phrases. Fiber-sum, geography problem, knot surgery, symplectic 4-manifolds.

This work was supported by Korea Research Foundation Grant (KRF-2004-013-C00002) and R14-2002-007-01002-0.

(C)2007 American Mathematical Society Reverts to public domain 28 years from publication 
Remarks 2. The key ingredient in the proof of Theorem 1.1 above is to use S. Boyer's result on simply connected 4-manifolds with a given boundary [B].

\section{The MAin CONSTRUCTION}

The main tactic in proving that many simply connected symplectic 4-manifolds admit infinitely many distinct smooth structures was to show that a family of simply connected homeomorphic 4-manifolds obtained by some topological surgeries have mutually different Seiberg-Witten invariants. We use the same idea in the nonsimply connected case. We first briefly review such topological surgeries - called a fiber sum and a knot surgery - and state related theorems.

Definition. For $i=1,2$, let $X_{i}$ be a symplectic 4-manifold containing a symplectic (or Lagrangian) genus $g$ surface $\Sigma_{g}$ of square zero. Suppose that $X_{i}^{0}=X_{i}-\nu_{i}\left(\Sigma_{g}\right)$ is a complement of a tubular neighborhood $\nu_{i}\left(\Sigma_{g}\right)$ of $\Sigma_{g}$ in $X_{i}$. Then, by choosing an orientation-reversing, fiber-preserving diffeomorphism $\varphi: \nu_{1}\left(\Sigma_{g}\right) \rightarrow \nu_{2}\left(\Sigma_{g}\right)$ and by gluing $X_{1}^{0}$ to $X_{2}^{0}$ along their boundaries via the diffeomorphism $\varphi \mid: \partial \nu_{1}\left(\Sigma_{g}\right) \rightarrow$ $\partial \nu_{2}\left(\Sigma_{g}\right)$, one can get a new symplectic 4-manifold $X_{1} \sharp_{\Sigma_{g}} X_{2}$, called a (symplectic) fiber sum of $X_{1}$ and $X_{2}$ along $\Sigma_{g}$.

Remarks 1. The Euler characteristic and the signature of a fiber sum 4-manifold $X_{1} \sharp_{\Sigma_{g}} X_{2}$ are easily computed, so that it has

$\chi\left(X_{1} \sharp \Sigma_{g} X_{2}\right)=\chi\left(X_{1}\right)+\chi\left(X_{2}\right)+(g-1)$ and $c_{1}^{2}\left(X_{1} \sharp_{\Sigma_{g}} X_{2}\right)=c_{1}^{2}\left(X_{1}\right)+c_{1}^{2}\left(X_{2}\right)+8(g-1)$.

Remarks 2. The minimality is preserved under a symplectic fiber sum operation. That is, if both $X_{1}$ and $X_{2}$ are minimal symplectic 4-manifolds, so is $X_{1} \sharp_{\Sigma_{g}} X_{2}$ (Theorem 2.5 in $[\mathrm{P} 1$ ).

Remarks 3. The Seiberg-Witten invariants of a fiber sum 4-manifold can be completely computed under certain cases. For example, the following product formula is widely known to the experts ([FS, $[\mathrm{Pd}]$ ).

Theorem 2.1 ( $\left[\mathrm{Pd}\right.$, Corollary 15 and Corollary 20). Suppose $X_{1}$ and $X_{2}$ are closed symplectic 4-manifolds which contain a symplectic torus $T$ in a cusp neighborhood. Then the Seiberg-Witten invariant of $X_{1} \sharp_{T} X_{2}$ is given by

$$
S W_{X_{1} \sharp T} X_{2}=S W_{X_{1}} \cdot S W_{X_{2}} \cdot(\exp ([T])-\exp (-[T]))^{2} .
$$

Definition. Suppose $K$ is a fibered knot in $S^{3}$. Let $M_{K}$ be a 3 -manifold obtained by performing 0 -framed surgery on $K$. Then the 3-manifold $M_{K}$ can be considered as a fiber bundle over circle, so that $M_{K} \times S^{1}$ fibers over torus $T$. If $X$ is a symplectic 4-manifold with a symplectically embedded torus $T$ of square 0 , then one can get a new symplectic 4-manifold $X_{K}:=X_{\sharp_{T}}\left(M_{K} \times S^{1}\right)$, obtained by taking a (symplectic) fiber sum along $T$; we call this a knot surgery with a knot $K$. R. Fintushel and R. Stern proved that $X_{K}$ is homotopy equivalent to $X$ under a mild condition on $X$ and they also computed the Seiberg-Witten invariant of $X_{K}$.

Theorem 2.2 ([FS). Suppose $X$ is a simply connected symplectic 4-manifold which contains a symplectic torus $T$ of square 0 in a cusp neighborhood with $\pi_{1}(X \backslash T)=$ 1. If $K$ is a fibered knot in $S^{3}$, then $X_{K}$ is a symplectic 4-manifold which is homeomorphic to $X$ and whose Seiberg-Witten invariant is

$$
S W_{X_{K}}=S W_{X} \cdot \Delta_{K}(t),
$$

where $\Delta_{K}(t)$ is the Alexander polynomial of a knot $K$ and $t=\exp (2[T])$. 
Next, we introduce some basic symplectic 4-manifolds which will serve as building blocks of our construction ([G], GS] for details).

Building Block 1. Let $Q:=Z_{1} \sharp_{\psi} Z_{2}$ be a symplectic 4-manifold constructed as follows: First, consider a Thurston's manifold $Z:=\mathbb{R}^{4} / G$, where $G$ is a discrete subgroup of symplectomorphisms generated by unit translations parallel to the $x^{1}-, x^{2}-$, and $x^{3}$-axes, together with the map $\left(x^{1}, \ldots, x^{4}\right) \mapsto\left(x^{1}+x^{2}, x^{2}, x^{3}, x^{4}+\right.$ 1). Note that projection onto the last two coordinates induces a bundle structure $\pi$ : $Z \rightarrow \mathbb{T}^{2}$ with symplectic torus fibers. Next, using two copies, $\pi_{i}: Z_{i} \rightarrow \mathbb{T}^{2}(i=1,2)$ of Thurston's manifold and using an orientation-reversing bundle map $\psi$ induced from $90^{\circ}$ rotation $\psi_{o}: \pi_{1}^{-1}(0) \rightarrow \pi_{2}^{-1}(0)$ defined by $\psi_{o}\left(x^{1}, x^{2}\right)=\left(-x^{2}, x^{1}\right)$, one obtains a symplectic fiber sum $Q:=Z_{1} \sharp_{\psi} Z_{2}$. This is a torus bundle over a genus 2 surface $\mathbb{T}^{2} \sharp \mathbb{T}^{2}$ and has a symplectic section $\Sigma_{2}$ of square 0 given by sections in $Z_{1}$ and $Z_{2}$ such that $\pi_{1}\left(Q-\Sigma_{2}\right) / \pi_{1}\left(\Sigma_{2}^{\prime \prime}\right)=1$, where $\Sigma_{2}^{\prime \prime}$ is a parallel copy of $\Sigma_{2}$. Similarly, there is also a Lagrangian torus $T \subset Q$ of square 0 , disjoint from $\Sigma_{2}$, in $Q$. For example, one obtains such a torus $T$ by setting $x^{1}=x^{4}=1 / 2$ in $Z_{1}$.

Building Block2. Let $E(n)$ be a simply connected elliptic surface with no multiple fibers and holomorphic Euler characteristic $n$. Then $E(n)$ can be obtained as an algebraic surface $B(2,3,6 n-1) \cup_{\Sigma(2,3,6 n-1)} C(n)$, where $B(2,3,6 n-1)$ is a Brieskorn manifold and $C(n)$, usually called a Gompf nucleus, is the neighborhood of a cusp fiber and a section which is an embedded 2 -sphere of square $-n$. By performing a knot surgery with a fibered knot $K \subset S^{3}$ in $C(n)$, one obtains a simply connected symplectic 4-manifold $E(n)_{K}$ which is homeomorphic, but not diffeomorphic, to $E(n)$. Since a Brieskorn manifold $B(2,3,6 n-1)$ with $n \geq 2$ contains a Lagrangian torus $T$ of square 0 , which intersects 2 -sphere transversely at a single point, lying in another Gompf nucleus, we conclude that $E(n)_{K}$ also contains a Lagrangian torus $T$ in a Gompf nucleus.

Using the building blocks above together with a Lefschetz fibration lying on the BMY-line [S], we constructed various families of simply connected, minimal, symplectic 4-manifolds which solve many geography problems in the simply connected case. Among them, we quote the following basic constructions.

Lemma 2.1 ( $\mathrm{P} 2$, Lemma 2.1). For each integer $k, 10 \leq k \leq 18$, there exists a simply connected, minimal, symplectic 4 -manifold $X_{3, k}$ with $\chi=2$ and $c_{1}^{2}=19-k$ which contains a symplectic genus 2 surface $\Sigma_{2}$ of square 0 and a symplectic torus $T$ of square 0 , disjoint from $\Sigma_{2}$, in a fishtail neighborhood, and $\pi_{1}\left(X_{3, k}-\Sigma_{2}\right)=$ $\pi_{1}\left(X_{3, k}-T\right)=1$.

Proposition 2.1 ([P3], Proposition 2.1). For each odd integer $m \geq 1$ and $10 \leq$ $k \leq 18$, there exists a simply connected, minimal, symplectic 4-manifold $Z_{m, k}$ which contains a symplectic genus 2 surface $\Sigma_{2}$ of square 0 and a torus $T$ of square 0 , disjoint from $\Sigma_{2}$, in a fishtail neighborhood which satisfies

$$
\pi_{1}\left(Z_{m, k}-\Sigma_{2}\right)=\pi_{1}\left(Z_{m, k}-T\right)=1 .
$$

Furthermore, it has $\chi\left(Z_{m, k}\right)=25 m^{2}+31 m+5$ and $c_{1}^{2}\left(Z_{m, k}\right)=225 m^{2}+248 m+$ $35-k$.

Building Block 3 . Let $G$ be a finitely presented group with $g$ generators $\left\{x_{1}, \ldots, x_{g}\right\}$ and $r$ relations $\left\{w_{1}, \ldots, w_{r}\right\}$. Let $F$ be an oriented genus $g$ Riemann surface with an oriented circles $\left\{\alpha_{1}, \ldots, \alpha_{g}, \beta_{1}, \ldots, \beta_{g}\right\}$ representing a standard symplectic basis 
for $H_{1}(F)$. For $i=1, \ldots, r$, let $\gamma_{i}$ be a smoothly immersed, oriented circle in $F$ representing the word $w_{i}$ in this group, and, for $i=1, \ldots, g$, let $\gamma_{r+i}=\beta_{i}$ and identifying $x_{i}=\alpha_{i}$, we have $\pi_{1}(F) /\left\langle\gamma_{1}, \ldots, \gamma_{g+r}\right\rangle \cong G$. By forming connected sums of $F$ with a copy of torus $T$ and perturbing and rearranging $\gamma_{i}$, R. Gompf constructed a collection of symplectic tori $T_{i}$ corresponding to $\gamma_{i} \times \alpha$ in $F \times \mathbb{T}$, where $\alpha$ is one of the oriented circles representing a standard basis of $H_{1}(\mathbb{T})$. Let $M=$ $(F \times \mathbb{T}) \sharp_{T_{i}} \cup_{i} E(1) \sharp_{\{\mathrm{p} t\} \times \mathbb{T}} E(1)$ be a symplectic 4 -manifold obtained by symplectically fiber summing $F \times \mathbb{T}$ with $E(1)$ 's along each $T_{i}$ and $\{\mathrm{p} t\} \times \mathbb{T}$. Then, since $E(1)-T$ is simply connected, $\pi_{1}(M) \cong G$. Furthermore, $M$ contains a symplectic torus $T=\left\{\mathrm{p} t^{\prime}\right\} \times \mathbb{T}$ such that $\pi_{1}(M) / \pi_{1}(T) \cong \pi_{1}(M)=G$. Recently, by modifying the construction above, S. Baldridge and P. Kirk constructed a similar symplectic 4-manifold with $\pi_{1}=G$.

Theorem 2.3 ([BK1], Theorem 6). Let $G$ have a presentation with g generators $\left\{x_{1}, \ldots, x_{g}\right\}$ and $r$ relations $\left\{w_{1}, \ldots, w_{r}\right\}$. Then there exists a closed symplectic 4-manifold $M$ with $\pi_{1}(M)=G$ which has $\chi(M)=g+r+1$ and $c_{1}^{2}(M)=0$.

Remark 1. The symplectic 4-manifold $M$ constructed in Theorem 2.3 above also contains a symplectic torus $T$ of square 0 such that the inclusion $i: T \rightarrow M$ induces a zero map $i_{*} \equiv 0: \pi_{1}(T) \rightarrow \pi_{1}(M)=G$. In fact, since $E(1)-T$ is simply connected, the inclusion $i: T^{\prime \prime} \rightarrow M-T$ also induces a zero map $i_{*} \equiv 0: \pi_{1}\left(T^{\prime \prime}\right) \rightarrow$ $\pi_{1}(M-T)$, where $T^{\prime \prime}$ is a parallel copy of $T$.

Suppose $X_{3, k}, Z_{m, k}$ and $M$ are symplectic 4-manifolds constructed in Lemma2.1, Proposition 2.1 and Theorem 2.3 respectively. Since $X_{3, k}$ contains a symplectic surface $\Sigma_{2}$ of square 0 , taking a fiber sum $l$ times along $\Sigma_{2}$ in $X_{3, k}$ with $l$ copies of $Q$, we get a symplectic 4-manifold $X_{3, k} \sharp_{\Sigma_{2}} l Q:=\left(\cdots\left(\left(X_{3, k} \sharp \Sigma_{2} Q\right) \sharp_{\Sigma_{2}} Q\right) \sharp_{\Sigma_{2}} \cdots \sharp_{\Sigma_{2}} Q\right)$. Again, since $Q$ contains a Lagrangian torus $T^{\prime}$ of square 0 disjoint from $\Sigma_{2}$, we can also construct a symplectic 4-manifold $\left(X_{3, k} \sharp_{\Sigma_{2}} l Q\right) \sharp_{T^{\prime}} E(2)$ by taking a fiber sum along $T^{\prime}$ in $Q$ and $C(2) \subset E(2)$. Furthermore, there is another symplectic torus $T$ lying in $M$ and $X_{3, k}-\Sigma_{2}$. Hence, by taking a fiber sum along $T$, we obtain a new symplectic 4-manifold

$$
X_{3, k, l}:=M \sharp_{T}\left(\left(X_{3, k} \sharp_{\Sigma_{2}} l Q\right) \sharp_{T^{\prime}} E(2)\right) \text {. }
$$

Similarly, using $Z_{m, k}$ instead of $X_{3, k}$, we obtain a symplectic 4-manifold

$$
Y_{m, k, l}:=M \sharp_{T}\left(\left(Z_{m, k} \sharp_{\Sigma_{2}} l Q\right) \sharp_{T^{\prime}} E(2)\right) \text {. }
$$

Then we have

Corollary 2.1. For each integer $k$ and $l, 10 \leq k \leq 18$ and $l \geq 0, X_{3, k, l}$ is a minimal symplectic 4-manifold with $\pi_{1}\left(X_{3, k, l}\right) \cong \pi_{1}(M)=G$ which satisfies $\chi\left(X_{3, k, l}\right)=l+g+r+5$ and $c_{1}^{2}\left(X_{3, k, l}\right)=8 l+19-k$.

Proof. It suffices to prove that $\pi_{1}\left(X_{3, k, l}\right) \cong \pi_{1}(M)$. For this, we first show that $\pi_{1}\left(\left(X_{3, k}-T\right) \sharp_{\Sigma_{2}} l Q\right)=1$ : Note that the Van-Kampen theorem on $\pi_{1}\left(X_{3, k}-T\right)=1$ implies that $\pi_{1}\left(\Sigma_{2}^{\prime \prime}\right) \rightarrow \pi_{1}\left(\left(X_{3, k}-T\right)-\Sigma_{2}\right)$ is a zero map and $\pi_{1}\left(\partial \nu\left(\Sigma_{2}\right)\right) \rightarrow$ $\pi_{1}\left(\left(X_{3, k}-T\right)-\Sigma_{2}\right)$ is surjective, where $\Sigma_{2}^{\prime \prime}$ is a parallel copy of $\Sigma_{2}$. Since $\pi_{1}\left(Q-\Sigma_{2}\right) / \pi_{1}\left(\Sigma_{2}^{\prime \prime}\right)=1$, we have

$$
\begin{aligned}
\pi_{1}\left(\left(X_{3, k}-T\right) \sharp_{\Sigma_{2}} Q\right) & =\pi_{1}\left(\left(X_{3, k}-T\right)-\nu\left(\Sigma_{2}\right)\right) * \pi_{1}\left(Q-\nu\left(\Sigma_{2}\right)\right) /\left\langle\pi_{1}\left(\partial \nu\left(\Sigma_{2}\right)\right)\right\rangle \\
& \cong \pi_{1}\left(\left(X_{3, k}-T\right)-\nu\left(\Sigma_{2}\right)\right) /\left\langle\pi_{1}\left(\partial \nu\left(\Sigma_{2}\right)\right)\right\rangle \\
& \cong 1
\end{aligned}
$$


Now, by induction on $l$, we conclude that $\pi_{1}\left(\left(X_{3, k}-T\right) \sharp_{\Sigma_{2}} l Q\right)=1$.

Next, since both $\left(X_{3, k}-T\right) \sharp_{\Sigma_{2}} l Q$ and $E(2)-T^{\prime}$ are simply connected, the fiber sum 4-manifold $\left(\left(X_{3, k}-T\right) \sharp_{\Sigma_{2}} l Q\right) \sharp_{T^{\prime}} E(2) \simeq\left(\left(X_{3, k}-\nu(T)\right) \sharp_{\Sigma_{2}} l Q\right) \sharp_{T^{\prime}} E(2)$ is also simply connected.

Finally, since the inclusion $i: T^{\prime \prime} \rightarrow M-T$ induces a zero map $i_{*} \equiv 0: \pi_{1}\left(T^{\prime \prime}\right) \rightarrow$ $\pi_{1}(M-T) \cong \pi_{1}(M-\nu(T))$ (see Remark11above), the Van-Kampen theorem implies that

$$
\begin{aligned}
\pi_{1}\left(X_{3, k, l}\right) & =\pi_{1}(M-\nu(T)) * \pi_{1}\left(\left(\left(X_{3, k} \sharp_{\Sigma_{2}} l Q\right) \sharp_{T^{\prime}} E(2)\right)-\nu(T)\right) /\left\langle\pi_{1}(\partial \nu(T))\right\rangle \\
& =\pi_{1}(M-\nu(T)) * \pi_{1}\left(\left(\left(X_{3, k}-\nu(T)\right) \sharp_{\Sigma_{2}} l Q\right) \sharp_{T^{\prime}} E(2)\right) /\left\langle\pi_{1}(\partial \nu(T))\right\rangle \\
& \cong \pi_{1}(M-\nu(T)) /\left\langle\pi_{1}(\partial \nu(T))\right\rangle \\
& \cong \pi_{1}(M-\nu(T)) * \pi_{1}(\nu(T)) /\left\langle\pi_{1}(\partial \nu(T))\right\rangle \\
& \cong \pi_{1}(M) .
\end{aligned}
$$

Corollary 2.2. For each odd integer $m \geq 1,10 \leq k \leq 18$ and $l \geq 0, Y_{m, k, l}$ is a minimal symplectic 4-manifold with $\pi_{1}\left(Y_{m, k, l}\right) \cong \pi_{1}(M)=G$ which satisfies $\chi\left(Y_{m, k, l}\right)=25 m^{2}+31 m+l+g+r+8$ and $c_{1}^{2}\left(Y_{m, k, l}\right)=225 m^{2}+248 m+8 l+35-k$.

Proof. Similar to the proof of Corollary 2.1 above, $\pi_{1}\left(Z_{m, k}-T\right)=1$ implies that $\pi_{1}\left(\left(\left(Z_{m, k}-\nu(T)\right) \sharp_{\Sigma_{2}} l Q\right) \sharp_{T^{\prime}} E(2)\right)=1$. Hence Van-Kampen theorem again induces

$$
\begin{aligned}
\pi_{1}\left(Y_{m, k, l}\right) & =\pi_{1}(M-\nu(T)) * \pi_{1}\left(\left(\left(Z_{m, k} \sharp_{\Sigma_{2}} l Q\right) \sharp_{T^{\prime}} E(2)\right)-\nu(T)\right) /\left\langle\pi_{1}(\partial \nu(T))\right\rangle \\
& =\pi_{1}(M-\nu(T)) * \pi_{1}\left(\left(\left(Z_{m, k}-\nu(T)\right) \sharp_{\Sigma_{2}} l Q\right) \sharp_{T^{\prime}} E(2)\right) /\left\langle\pi_{1}(\partial \nu(T))\right\rangle \\
& \cong \pi_{1}(M-\nu(T)) /\left\langle\pi_{1}(\partial \nu(T))\right\rangle \\
& \cong \pi_{1}(M) .
\end{aligned}
$$

Next, we introduce S. Boyer's result on simply connected 4-manifolds with a given boundary $[\mathrm{B}$, which will be a key ingredient in proving our result.

Let $V_{1}$ and $V_{2}$ be simply connected, compact, oriented 4-manifolds with connected boundary $\partial V_{1}=\partial V_{2}$ and suppose that $f: \partial V_{1} \rightarrow \partial V_{2}$ is an orientation preserving homeomorphism. Then there are two obstructions to extending $f$ to a homeomorphism $F: V_{1} \rightarrow V_{2}$ : The first is to find an isometry $\Lambda:\left(H_{2}\left(V_{1} ; \mathbf{Z}\right), Q_{V_{1}}\right) \rightarrow$ $\left(H_{2}\left(V_{2} ; \mathbf{Z}\right), Q_{V_{2}}\right)$ for which the following diagram commutes:

$$
\begin{aligned}
& 0 \longrightarrow H_{2}\left(\partial V_{1}\right) \longrightarrow H_{2}\left(V_{1}\right) \longrightarrow H_{2}\left(V_{1}, \partial V_{1}\right) \longrightarrow H_{1}\left(\partial V_{1}\right) \longrightarrow 0 \\
& \downarrow f_{*} \quad \downarrow \Lambda \quad \downarrow \Lambda^{*} \quad \downarrow f_{*} \\
& 0 \longrightarrow H_{2}\left(\partial V_{2}\right) \longrightarrow H_{2}\left(V_{2}\right) \longrightarrow H_{2}\left(V_{2}, \partial V_{2}\right) \longrightarrow H_{1}\left(\partial V_{2}\right) \longrightarrow 0
\end{aligned}
$$

Here an isometry means an isomorphism $\Lambda: H_{2}\left(V_{1} ; \mathbf{Z}\right) \rightarrow H_{2}\left(V_{2} ; \mathbf{Z}\right)$ which preserves an intersection form $Q_{V_{i}}$, and $\Lambda^{*}$ is the adjoint of $\Lambda$ with respect to the identification of $H_{2}\left(V_{i}, \partial V_{i}\right)$ with $\operatorname{Hom}\left(H_{2}\left(V_{i}\right) ; \mathbf{Z}\right)$ arising from Lefschetz duality. We call such a pair $(f, \Lambda)$ a morphism and denote it symbolically as $(f, \Lambda): V_{1} \rightarrow V_{2}$. The second obstruction encountered is to realize a given morphism $(f, \Lambda)$ geometrically, i.e. to find a homeomorphism $F: V_{1} \rightarrow V_{2}$ such that $(f, \Lambda)=\left(\left.F\right|_{\partial V_{1}}, F_{*}\right)$. $\mathrm{S}$. Boyer showed when a given morphism could be realized geometrically. Explicitly, he proved

Theorem 2.4 ( $\left[\mathrm{B}\right.$, Theorem 0.7 and Proposition 0.8). If $(f, \Lambda): V_{1} \rightarrow V_{2}$ is a morphism between two simply connected smooth 4-manifolds $V_{1}$ and $V_{2}$ with boundary $\partial V_{1}=\partial V_{2}$, there is an obstruction $\theta(f, \Lambda) \in I^{1}\left(\partial V_{1}\right)$ such that $(f, \Lambda)$ is realized 
geometrically if and only if $\theta(f, \Lambda)=0$. Furthermore, if $H_{1}\left(\partial V_{1} ; \mathbf{Q}\right)=0$, then $\theta(f, \Lambda)=0$.

Corollary 2.3. Suppose that $\partial V_{1}=\partial V_{2}$ is a homology 3-sphere. Then, for any morphism $(f, \Lambda): V_{1} \rightarrow V_{2}$ between two simply connected smooth 4-manifolds $V_{1}$ and $V_{2}$, there is a homeomorphism $F: V_{1} \rightarrow V_{2}$ such that $(f, \Lambda)=\left(\left.F\right|_{\partial V_{1}}, F_{*}\right)$.

Finally, we state and prove a key proposition.

Proposition 2.2. Suppose $X$ is a symplectic 4-manifold which contains a symplectic torus $T$ lying in a cusp neighborhood. Then, for each integer $n \geq 2$, a family of symplectic 4-manifolds $\left\{X \sharp_{T} E(n)_{K} \mid K\right.$ is a fibered knot in $\left.S^{3}\right\}$ are mutually non-diffeomorphic, but all homeomorphic. Furthermore, $\pi_{1}\left(X \sharp_{T} E(n)_{K}\right) \cong \pi_{1}(X)$.

Proof. Since $E(n)_{K}$ contains a Gompf nucleus, say $C_{n}$, embedded in $B(2,3,6 n-1)$ (Building Block 2), we can decompose $E(n)_{K}$ into $C_{n} \cup_{\Sigma} E(n)_{K}^{\circ}$, where $\Sigma$ is a homology 3-sphere and $E(n)_{K}^{\circ}$ denotes a complement of $C_{n}$ in $E(n)_{K}$. Note that the Gompf nucleus $C_{n}$ is independent of $K$, i.e. $C_{n}$ is a common submanifold of codimension zero in all $E(n)_{K}$ 's. Hence, after taking a symplectic fiber sum of $X$ with $E(n)_{K}$ along a Lagrangian torus $T \subset C_{n}$, we get a decomposition

$$
X \sharp_{T} E(n)_{K}=\left(X \sharp_{T} C_{n}\right) \cup_{\Sigma} E(n)_{K}^{\circ} \text {. }
$$

In order to show that $X \sharp_{T} E(n)_{K}$ is homeomorphic to $X \sharp_{T} E(n)_{K^{\prime}}$ for any fibered knots $K$ and $K^{\prime}$ in $S^{3}$, we first choose an identity map $f=i d: X \sharp_{T} C_{n} \rightarrow X \sharp_{T} C_{n}$ and an isomorphism $\Lambda: H_{2}\left(E(n)_{K}^{\circ} ; \mathbf{Z}\right) \rightarrow H_{2}\left(E(n)_{K^{\prime}}^{\circ} ; \mathbf{Z}\right)$. In particular, since both intersection forms $Q_{E(n)}{ }_{K}^{\circ}$ and $Q_{E(n)_{K}^{\circ}}^{\circ}$ are unimodular and indefinite, they are uniquely determined by rank, signature and type, so that they are isomorphic. (The reason is following: Since $E(n)_{K}$ and $E(n)_{K^{\prime}}$ are homeomorphic, their intersection forms have the same rank, signature and type. Hence $Q_{E(n)_{K}^{\circ}}$ and $Q_{E(n)_{K}^{\prime}}$ also have the same rank, signature and type.) Hence we may choose $\Lambda$ to be an isometry, i.e. $\Lambda:\left(H_{2}\left(E(n)_{K}^{\circ} ; Q_{E(n)}^{\circ}\right) \rightarrow\left(H_{2}\left(E(n)_{K^{\prime}}^{\circ} ; Q_{E(n)_{K^{\prime}}^{\circ}}\right)\right.\right.$ is an isomorphism which preserves the intersection forms. Then, applying Corollary 2.3 with the fact that $\Sigma$ is a homology 3 -sphere, we conclude that there exists a homeomorphism $F$ : $E(n)_{K}^{\circ} \rightarrow E(n)_{K^{\prime}}^{\circ}$ such that $(f, \Lambda)=\left(\left.F\right|_{\Sigma}, F_{*}\right)$. Thus there is a homeomorphism between two symplectic 4-manifolds $X \sharp_{T} E(n)_{K}$ and $X \sharp_{T} E(n)_{K^{\prime}}$.

Next, applying Theorem 2.1 and Theorem 2.2 above, we also conclude that $X \sharp_{T} E(n)_{K}$ and $X \sharp_{T} E(n)_{K^{\prime}}$ have different Seiberg-Witten invariants as long as $\Delta_{K}(t) \neq \Delta_{K^{\prime}}(t)$, i.e. $X \sharp_{T} E(n)_{K}$ is not diffeomorphic to $X \sharp_{T} E(n)_{K^{\prime}}$.

The last statement follows from the Van-Kampen theorem using the fact that $T$ is a regular fiber in a cusp neighborhood and $\pi_{1}\left(E(n)_{K}-T\right)=1$ :

$$
\begin{aligned}
\pi_{1}\left(X \sharp_{T} E(n)_{K}\right) & =\pi_{1}(X-\nu(T)) * \pi_{1}\left(E(n)_{K}-\nu(T)\right) /\left\langle\pi_{1}(\partial \nu(T))\right\rangle \\
& \cong \pi_{1}(X-\nu(T)) /\left\langle\pi_{1}(\partial \nu(T))\right\rangle \\
& \cong \pi_{1}(X-\nu(T)) * \pi_{1}(\nu(T)) /\left\langle\pi_{1}(\partial \nu(T))\right\rangle \\
& \cong \pi_{1}(X) .
\end{aligned}
$$

Proof of Theorem 1.1. Let $\left\{X_{3, k, l}\right\}$ and $\left\{Y_{m, k, l}\right\}$ be minimal symplectic 4-manifolds appeared in Corollary 2.1 and Corollary 2.2 above. For each odd integer $m \geq 1$ with a finitely presented group $G$ fixed (so that $g$ and $r$ are also fixed), define two 
numbers $r_{G, m}$ and $t_{G, m}$ by

$$
\left\{\begin{aligned}
r_{G, m} & :=\frac{c_{1}^{2}\left(Y_{m, 10,112.5 m+173.5}\right)}{\chi\left(Y_{m, 10,112.5 m+173.5}\right)}=\frac{225 m^{2}+1148 m+1413}{25 m^{2}+143.5 m+181.5+g+r}, \\
t_{G, m} & :=25 m^{2}+143.5 m+181.5+g+r .
\end{aligned}\right.
$$

Then $\left\{r_{G, m}\right\}$ is an increasing sequence converging to 9 and any lattice point $(x, \mathbf{c})$ satisfying $0 \leq \mathbf{c} \leq r_{G, m} x$ and $x \geq t_{G, m}$ is realized as chern numbers $\left(\chi, c_{1}^{2}\right)$ of a minimal symplectic 4 -manifold

$$
X_{3, k, l \sharp_{T}} E(n)_{K} \quad \text { or } \quad Y_{m, k^{\prime}, l^{\prime} \sharp_{T}} E(n)_{K}
$$

for some integers $m, k, k^{\prime}, l, l^{\prime}$ and $n$. Finally, since $X_{3, k, l}$ and $Y_{m, k, l}$ contain a symplectic torus $T$ in a Gompf nucleus $\subset E(2)$, we conclude from Proposition 2.2 above that both $X_{3, k, l} \sharp_{T} E(n)_{K}$ and $Y_{m, k, l} \sharp_{T} E(n)_{K}$ admit infinitely many distinct smooth structures and they have $\pi_{1}=G$.

\section{REFERENCES}

[BK1] S. Baldridge and P. Kirk, On symplectic 4-manifolds with prescribed fundamental group, to appear in Commentarii Math. Helv. 82 (2007)

[BK2] S. Baldridge and P. Kirk, Symplectic 4-manifolds with arbitrary fundamental group near the Bogomolov-Miyaoka-Yau line, to appear in Jour. of Symplectic Geometry 4 (2006)

[B] S. Boyer, Simply connected 4-manifolds with a given boundary, Trans. Amer. Math. Soc. 298 (1986), 331-357 MR0857447 (88b:57023)

[FS] R. Fintushel and R. Stern, Knots, links and 4-manifolds, Invent. Math. 134 (1998), 363400 MR:1650308 (99j:57033)

[G] R. Gompf, A new construction of symplectic manifolds, Annals of Math. 142 (1995), 527-595 MR1356781 (96j:57025)

[GS] R. Gompf and A. Stipsicz, 4-manifolds and Kirby calculus, Graduate Studies in Mathematics 20 (1999), AMS MR1707327 (2000h:57038)

[Pd] D. Park, A gluing formula for the Seiberg-Witten invariant along $T^{3}$, Michigam Math. Jour. 50 (2002), 593-611 MR1935154(2003i:57051)

[P1] J. Park, The geography of irreducible 4-manifolds, Proc. Amer. Math. Soc 126 (1998), 2493-2503 MR1487335 (98j:57034)

[P2] J. Park, Exotic smooth structures on 4-manifolds, Forum Math. 14 (2002), 915-929 MR.1932526 (2003i:57046)

[P3] J. Park, Exotic smooth structures on 4-manifolds II, Topology and its Appl. 132 (2003), 195-202 MR.1991809 (2004d:57033)

[S] A. Stipsicz, Simply connected symplectic 4-manifolds with positive signature, Turkish J. Math. 23 (1999), 145-150 MR1701643(2001e:57029)

[T] C. Taubes, The Seiberg-Witten invariants and symplectic forms, Math. Res. Lett. 1 (1994), 809-822 MR 1306023 (95j:57039)

Department of Mathematical Sciences, Seoul National University, San 56-1 SillimDong, GwanaK-Gu, Seoul 151-747, Korea

E-mail address: jipark@math.snu.ac.kr 